\title{
Inverse design engineering of all-silicon polarization beam splitters
}

\author{
Frandsen, Lars Hagedorn; Sigmund, Ole
}

Published in:

Proceedings of SPIE

Link to article, DOI:

$10.1117 / 12.2210848$

Publication date:

2016

Document Version

Publisher's PDF, also known as Version of record

Link back to DTU Orbit

Citation (APA):

Frandsen, L. H., \& Sigmund, O. (2016). Inverse design engineering of all-silicon polarization beam splitters. In A. Adibi, S-Y. Lin, \& A. Scherer (Eds.), Proceedings of SPIE: Photonic and Phononic Properties of Engineered Nanostructures VI (Vol. 9756). [97560Y] SPIE - International Society for Optical Engineering. Proceedings of SPIE - The International Society for Optical Engineering https://doi.org/10.1117/12.2210848

\section{General rights}

Copyright and moral rights for the publications made accessible in the public portal are retained by the authors and/or other copyright owners and it is a condition of accessing publications that users recognise and abide by the legal requirements associated with these rights.

- Users may download and print one copy of any publication from the public portal for the purpose of private study or research.

- You may not further distribute the material or use it for any profit-making activity or commercial gain

- You may freely distribute the URL identifying the publication in the public portal 


\title{
Inverse design engineering of all-silicon polarization beam splitters
}

\author{
Lars H. Frandsen ${ }^{\mathrm{a}}$, Ole Sigmund ${ }^{\mathrm{b}}$ \\ ${ }^{a}$ DTU Fotonik, Department of Photonics Engineering, Technical University of Denmark \\ ${ }^{b}$ DTU Mekanik, Department of Mechanics Engineering, Technical University of Denmark \\ 2800 Lyngby, Denmark
}

\begin{abstract}
Utilizing the inverse design engineering method of topology optimization, we have realized high-performing all-silicon ultra-compact polarization beam splitters. We show that the device footprint of the polarization beam splitter can be as compact as $\sim 2 \mu \mathrm{m}^{2}$ while performing experimentally with a polarization splitting loss lower than $\sim 0.82 \mathrm{~dB}$ and an extinction ratio larger than $\sim 15 \mathrm{~dB}$ in the C-band. We investigate the device performance as a function of the device length and find a lower length above which the performance only increases incrementally. Imposing a minimum feature size constraint in the optimization is shown to affect the performance negatively and reveals the necessity for light to scatter on a sub-wavelength scale to obtain functionalities in compact photonic devices.
\end{abstract}

Keywords: Silicon photonics, inverse design engineering, topology optimization, polarization beam splitter.

\section{INTRODUCTION}

On the venture to reduce the size of integrated optical devices while keeping a high level of performance, inverse design engineering (iDE) has shown to be an attractive concept to remove geometrical restrictions and to allow a larger parameter space to be included in the design phase. This will pave the way for objective-driven and optimal design solutions [1-4] with ultra-small footprints in nanophotonic circuits. Specifically, topology optimization (TopOpt) [1,5] has been experimentally proven to be a strong and robust iDE tool for designing various low-loss and ultra-compact nanophotonic components with wavelength or mode handling properties [1,6-10]. Meanwhile, polarization-handling devices play a pivotal role in high-index contrast nanophotonic devices because of an inherent strong birefringence [11], which leads to polarization-sensitive performances of devices. Thus, optical signals travelling with different polarization states will need to be processed by separate nanophotonic components and requires an efficient, broadband, and ultracompact polarization beam splitter (PBS) to be available for the design of the nanophotonic circuit. Several design approaches have been taken to achieve ultra-compact PBS designs including utilizing a silicon hybrid plasmonic waveguide [12], an objective-first iDE method [2], and a digital nanopatterning iDE method [4]. All PBS designs in [2, $4,12]$ have compact footprints of $\sim 5-8 \mu^{2}$. A thorough investigation of the capabilities, tradeoffs, and performances of the different approaches in order to provide a most-compact and best-performing PBS design lacks and is also not the purpose of this paper. In this paper, we demonstrate the capability for 3D TopOpt to handle and optimize polarization selective functionalities in a nanophotonic device as we design and optimize functional PBS designs with $\mu \mathrm{m}^{2}$-sized footprints. The obtained performances for different device- and filter-sizes utilized in the TopOpt are calculated. We verify the performance of the TopOpt method experimentally by fabricating a PBS with a device footprint of $\sim 2 \mu \mathrm{m}^{2}$ in silicon-on-insulator material.

\section{DESIGN \& OPTIMIZATION}

\subsection{A $2 \mu \mathrm{m}^{2}$ polarization beam splitter}

The initial and naïve design of our PBS is shown in Fig. 1(a) and is comprised of óne input and two output silicon singlemode waveguides of width $0.44 \mu \mathrm{m}$ that support the fundamental transverse-electric (TE) and -magnetic (TM) mode. The waveguides connect to a $1.4 \mu \mathrm{m} \times 1.4 \mu \mathrm{m}$ (length $\mathrm{x}$ height) multi-mode interferometer (MMI) block (solid orange), which is the target area for the PBS functionality. For comparison, the size of the device in e.g. [4] is $2.4 \mu \mathrm{m} \times 2.4 \mu \mathrm{m}$. We choose to align the input port vertically to the lower output port targeted for the TM mode. This is done as initial investigations of the MMI structure reveal that the TE mode will diffract more in the MMI section compared to the TM mode. In this way, a better performance of the optimized PBS is expected by having an initial structure promoting this attribute and allowing the TopOpt procedure to exploit it more easily.

Photonic and Phononic Properties of Engineered Nanostructures VI, edited by Ali Adibi, Shawn-Yu Lin, Axel Scherer, Proc. of SPIE Vol. 9756, 97560Y · @ 2016 SPIE · CCC code: 0277-786X/16/\$18 · doi: 10.1117/12.2210848 


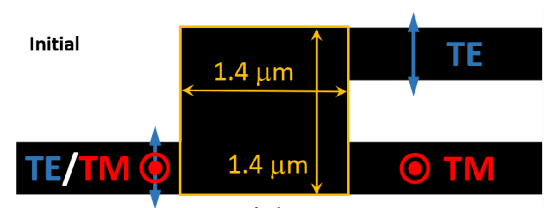

(a)

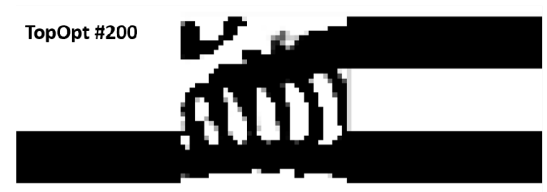

(d)

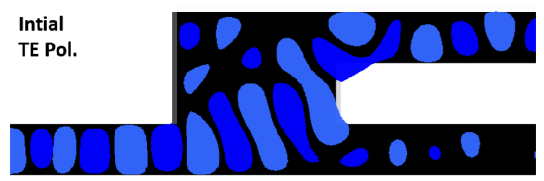

(b)

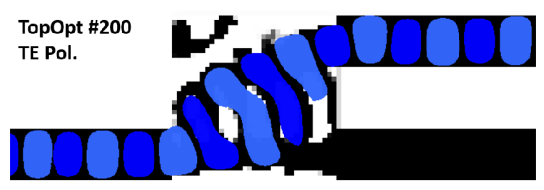

(e)

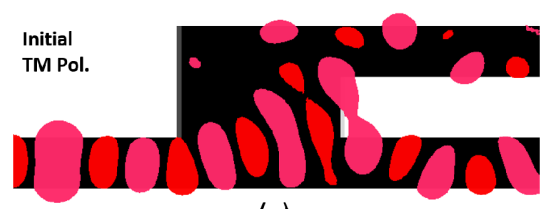

(c)

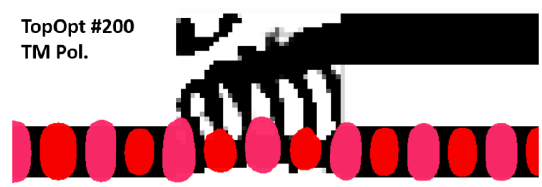

(f)

Figure 1. (a) The input MMI structure for the 3D TopOpt procedure where TE (blue)/TM (red) polarized input light is targeted to the upper/lower $0.44 \mu \mathrm{m}$ wide output port, respectively. The size of the device is $1.4 \mu \mathrm{m} \times 1.4 \mu \mathrm{m}$ (solid orange). (b, c) The calculated out-of-plane component of the magnetic (b) and electric (c) field plots when inputting TE (b) and TM (c) polarized light at $1550 \mathrm{~nm}$ to the initial structure. (d) The topology optimized design of the $1.4 \mu \mathrm{m} \times 1.4 \mu \mathrm{m}$ silicon PBS achieved after 200 iterations steps. (e, f) The calculated out-of-plane component of the magnetic (e) and electric (f) field plots when inputting TE (e) and TM (f) polarized light at $1550 \mathrm{~nm}$ to the optimized structure.

Figure 1(b) and 1(c) show the calculated out-of-plane component of the magnetic / electric field plots when inputting TE- / TM-polarized light at $1550 \mathrm{~nm}$ into the initial design, respectively. It can be seen that TE-polarized light is directed more towards the upper output port, while TM-polarized light is mainly transmitted to the lower arm. Hence, the objective of the TopOpt procedure is to optimize the demultiplexing of the TE mode from the input port to the upper output port while the TM mode should transmit to the lower output port in the full $\sim 300$ nm wavelength range of the input signal, which is centered on $1600 \mathrm{~nm}$. In the 3D optimization, the thickness of the silicon device slab with relative permittivity $\varepsilon_{\mathrm{si}}=11.68$ is $0.34 \mu \mathrm{m}$ and has air above and a silica buffer with relative permittivity $\varepsilon_{\text {silica }}=2.085$ below. The design domain for the TopOpt is the $1.4 \mu \mathrm{m}$ x $1.4 \mu \mathrm{m}$ MMI block and it extends vertically through the silicon device slab. The designed structures are constrained to be uniform in the vertical direction to make the optimized designs feasible for fabrication using a conventional dry etching technique. Topology optimization is performed utilizing 3D finite-difference time-domain (FDTD) modeling with a $40 \mathrm{~nm}$ spatial resolution, a total of 300000 mesh cells, and perfectly matched absorbing layers as boundary conditions using a software package developed in-house [13]. The optimization process is based on repeated solutions of the state and adjoint equations combined with mathematical programming-based design updates [14]. The TopOpt was run for 200 iteration steps on a computer cluster with 120 Intel Xeon E5-2660 cores distributed on 6 nodes connected with InfiniBand. The total computation time was $\sim 12$ hours. Figure 1(d) shows the optimized and converged PBS design achieved after 200 iteration steps. Clearly, a grating-like structure has been introduced in the MMI block. The field plots of TE- and TM- polarized light at $1550 \mathrm{~nm}$ propagated through the optimized structure are shown in Fig. 1(e) and Fig. 1(f), respectively, and reveal that the grating-like structure acts on the TE-polarized light so that it is diffracted to the upper output port while TM-polarized light is transmitted unaffected to the lower output port. Hence, the topology optimized PBS operates as required.

\subsection{Device size}

We investigate the dependency of the PBS performance on the device size by optimizing PBSs with different lengths of $1.0,1.4,1.8,2.2$, and $2.6 \mu \mathrm{m}$ while keeping the height fixed to $1.4 \mu \mathrm{m}$. Here, only the length is varied as we from the previous section expect the optimized structures to have a grating-like structure, i.e. we are investigating the influence of the length of the topology-optimized grating. Figure 2 shows the normalized transmission spectra for (a) TE- / (c) TMpolarized light in the upper/lower output port, respectively. These are calculated for the devices shown in the left of Fig. 2 (color codes apply). Specifically, the $1.4 \mu \mathrm{m} \times 1.4 \mu \mathrm{m}$ (pink) device from the previous section performs numerically in the $300 \mathrm{~nm}$ bandwidth with an insertion loss lower than $0.54 \mathrm{~dB} / 0.65 \mathrm{~dB}$ for TE / TM polarized light demultiplexed to the upper / lower output port, respectively. Figure 2(b) and 2(d) show the corresponding polarization extinction ratio (PER), which for the $1.4 \mu \mathrm{m} \times 1.4 \mu \mathrm{m}$ (pink) PBS are better than $15 \mathrm{~dB}$. By reducing the length to $1.0 \mu \mathrm{m}$, we see a considerably degradation of the performance increasing the insertion loss to $\sim 1.1 \mathrm{~dB}$ and lowering the PER to $\sim 12 \mathrm{~dB}$. On the other hand, increasing the length of the device in steps of $0.4 \mu \mathrm{m}$ gradually improves the performance. The longest device with a footprint of $2.6 \mu \mathrm{m} \times 1.4 \mu \mathrm{m}\left(3.64 \mu \mathrm{m}^{2}\right)$ has an insertion loss lower than $0.3 \mathrm{~dB} / 0.5 \mathrm{~dB}$ for the TE 
/ TM mode while keeping the PERs better than $19 \mathrm{~dB} / 17 \mathrm{~dB}$, respectively. Limiting the operation to the C-band the numbers are even better with demultiplexing losses around $0.15 \mathrm{~dB}$ and PERs larger than $24 \mathrm{~dB}$. Further investigations could include extending the length and height of the device.
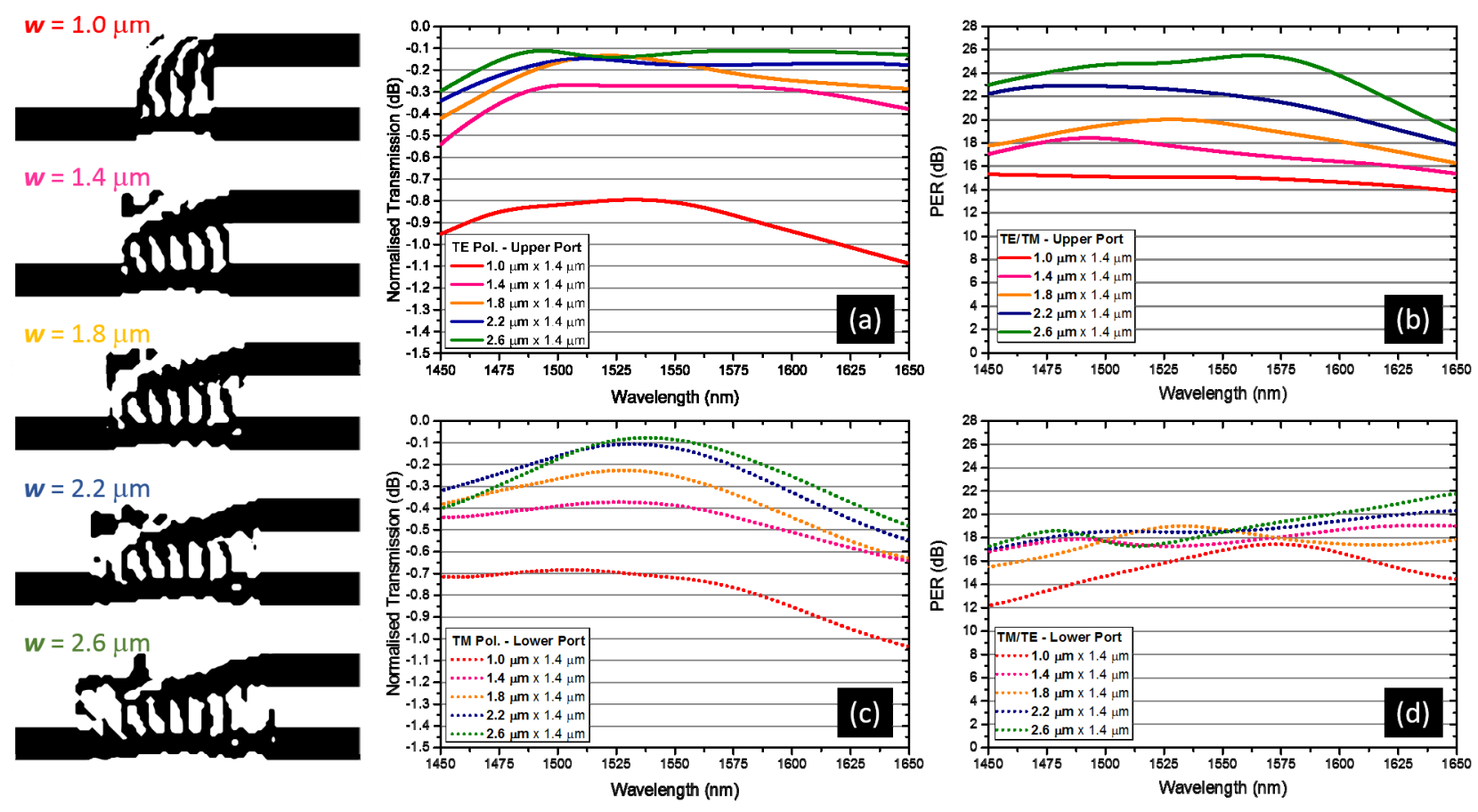

Figure 2. Topology optimized PBS designs with lengths of $1.0 \mu \mathrm{m}$ (red), $1.4 \mu \mathrm{m}$ (pink), $1.8 \mu \mathrm{m}$ (orange), $2.2 \mu \mathrm{m}$ (blue), and $2.6 \mu \mathrm{m}$ (green). (a, c) Normalized transmission spectra for the (a) TE- and (c) TM-polarized mode recorded in the (a) upper and (c) lower output port of the PBSs shown to the left (color codes apply). (b, d) The corresponding ERs.

\subsection{Feature size}

Inverse design engineering methods typically leads to complex and subwavelength nanostructurings of the photonic devices to fulfill the target objectives. In this way, the optimized structures set tough requirements to the fabrication procedure, which normally requires state-of-the-art electron-beam lithography and is not compatible with massfabrication technologies such as deep-UV lithography. Hence, for iDE to be of practical interest for photonic integration in a not-so-distant future, the dependency of the device performance on the feature sizes introduced in the iDE should be investigated. In order to do so, we have applied TopOpt to identical PBS design problems using filters of different sizes [14] in the TopOpt procedure. The mesh size and the objectives are kept the same for all optimizations, which are applied to identical starting designs with footprints of $2.6 \mu \mathrm{m} \times 1.4 \mu \mathrm{m}$. Figure 3(a) shows the obtained designs achieved using filters of sizes F1=40 nm (green), F2=120 nm (blue), and F3=160 nm (red). The effect of increasing the filter size is clearly seen in Fig. 3(a) where features in the grating-like structure are removed when increasing the filter size. For the largest filter size used here, all what remains in the design are two relatively smooth and coupled waveguides. The normalized transmission spectra and the PERs of the PBSs are shown in Fig. 3(b) and Fig. 3(c), respectively. All three PBSs keep their insertion losses below $3 \mathrm{~dB}$ in the $200 \mathrm{~nm}$ bandwidth, however, the PERs drop from $\sim 17 \mathrm{~dB}$ for the smallest $40 \mathrm{~nm}$ filter to $\sim 6 \mathrm{~dB}$ for the largest $160 \mathrm{~nm}$ filter. Hence, not surprisingly, filtering out smaller features during the optimization while keeping the mesh and design sizes fixed results in lower performance of the PBS. This reveals the power that lies in letting light scatter on a sub-wavelength scale. Further investigations with respect to feature sizes will include enlarging the footprint of the initial design when applying larger filter sizes. 


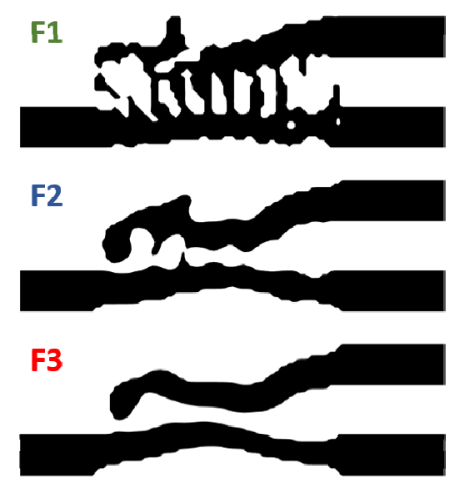

(a)

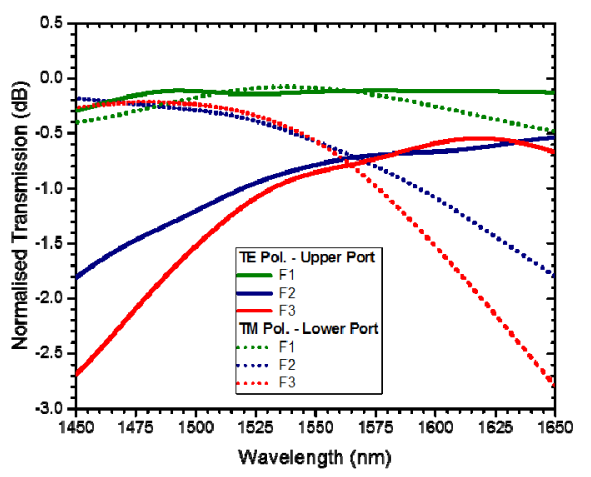

(b)

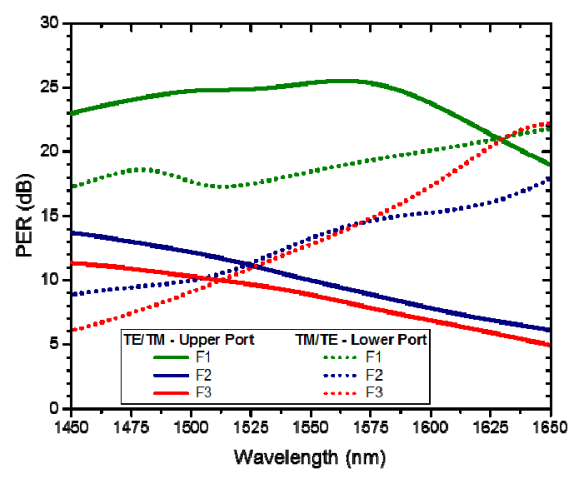

(c)

Figure 3. Topology optimized PBS designs with length of $2.6 \mu \mathrm{m}$ and optimized utilizing different filters sizes $\mathrm{F} 1=40 \mathrm{~nm}$ (green), F2=120 nm (blue), and F3=160 nm (red). (b) Normalized transmission spectra for the (solid) TE- and (dotted) TMpolarized mode recorded in the (solid) upper and (dotted) lower output port of the PBSs shown to the left (color codes apply). (c) The corresponding PERs.

\section{FABRICATION \& CHARACTERIZATION}

We have fabricated the PBS design with a footprint of $1.4 \mu \mathrm{m} \times 1.4 \mu \mathrm{m}\left(\sim 2 \mu \mathrm{m}^{2}\right)$ shown in Fig. 4(a) in silicon-oninsulator (SOI) material utilizing electron-beam lithography. The topology-optimized design was defined in an $\sim 110 \mathrm{~nm}$ thick ZEP520A electron beam resist by a JEOL JBX-9500 electron-beam lithography system operating at $100 \mathrm{keV}$ with a writing field of $1 \mathrm{~mm} \times 1 \mathrm{~mm}$. The electron beam with an estimated size of $12 \mathrm{~nm}$ is scanned in steps of $4 \mathrm{~nm}$ and proximity correction is applied to take backscattered electrons into account. After development, the electron beam resist is used as a soft mask in an inductively coupled plasma reactive ion etch utilizing $\mathrm{SF}_{6}$ and $\mathrm{C}_{4} \mathrm{~F}_{8}$ gases to transfer the patterns into the top $340 \mathrm{~nm}$ silicon layer of a SOI wafer having a $3000 \mathrm{~nm}$ buffer layer of silica. Inversely tapered silicon ridge- and partly overlapping $\sim 3 \mu \mathrm{m} \times \sim 3 \mu \mathrm{m}$ SU-8 polymer-waveguides are used to improve coupling to/from tapered and lensed single-mode fibers. Figure 4(b) shows the scanning electron micrograph (SEM) image of the fabricated PBS. Comparing the designed and the fabricated PBS, we see a high quality of the fabrication, where only a slight smoothening of the smallest features has occurred.

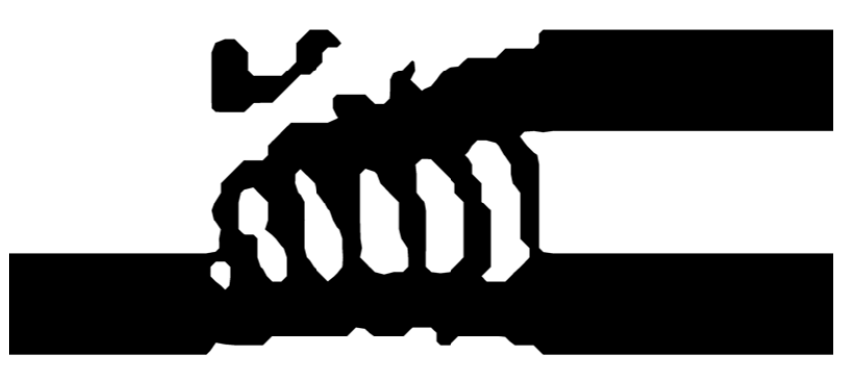

(a)

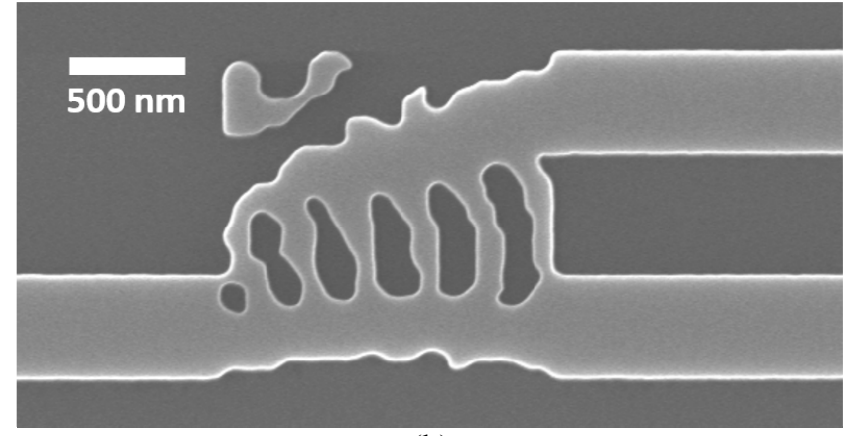

(b)

Figure 4. (a) The topology optimized design of the $1.4 \mu \mathrm{m} \times 1.4 \mu \mathrm{m}$ silicon PBS. (b) SEM image of the PBS fabricated in silicon-on-insulator material.

The fabricated PBS was characterized optically using a laser source tunable from $1520 \mathrm{~nm}$ to $1620 \mathrm{~nm}$ and transmission spectra were recorded on an optical spectrum analyzer. The polarization of the incoming light was established with an extinction ratio $\sim 20-25 \mathrm{~dB}$ by using a polarization controller before the chip and a polarization controller and a polarizer after the chip similar to the procedure described in [4]. Light was coupled to/from inversely tapered waveguides on the chip by using tapered lensed fibers. 


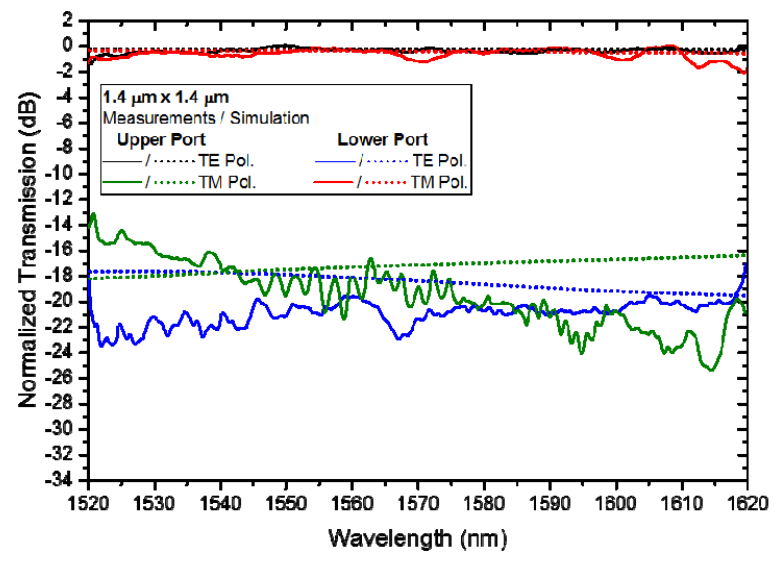

(a)

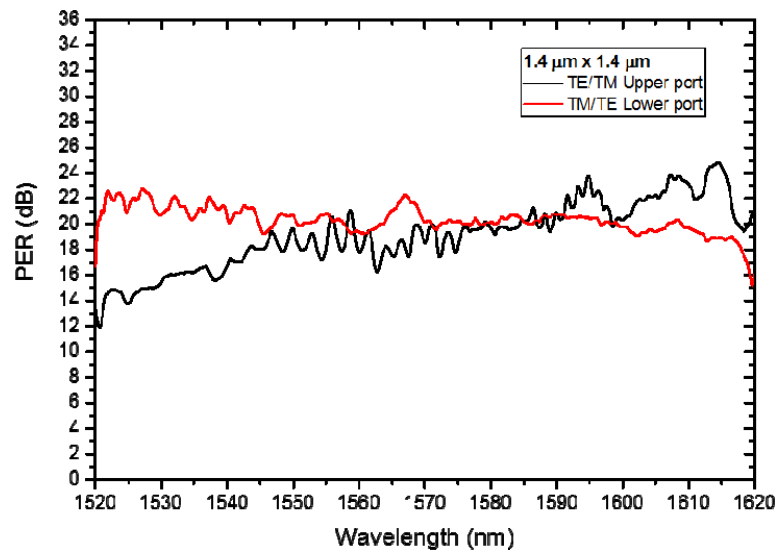

(b)

Figure 5. Measured (solid) and 3D-FDTD calculated (dotted) transmission spectra for TE (black/blue) and TM (red/green) polarized light in the upper (black/red) and lower (blue/green) output port. All spectra have been normalized to the transmission through a waveguide of similar length. (b) The corresponding PERs for TE (black) and TM (red) in the upper and lower output port, respectively.

Figure 5 shows the measured spectra (solid) for (black/blue) TE- and (red/green) TM- polarized light de-multiplexed to the upper (black/green) and lower (red/blue) output port, respectively. The corresponding 3D FDTD-calculated spectra (dotted) of the PBS are also shown and nicely predicts the measured spectra for the transmitted polarizations (black and red). The discrepancies between the measured and the calculated spectra for the rejected polarizations (blue and green) are attributed to the smoothened features in the fabricated design. For the TE / TM polarization, we experimentally find an insertion loss lower than $\sim 0.82 \mathrm{~dB} / \sim 2.1 \mathrm{~dB}$ with PERs better than $\sim 12 / \sim 15 \mathrm{~dB}$, respectively, in the $100 \mathrm{~nm}$ bandwidth limited by the tunable laser. In the C-band, the insertion losses are lower than $\sim 0.82 \mathrm{~dB}$ and the PERs are better than $\sim 15 \mathrm{~dB}$ for both polarization modes. For comparison, the PBS in [4] with a footprint of $2.4 \mu \mathrm{m} \times 2.4 \mu \mathrm{m}$ $\left(5.76 \mu \mathrm{m}^{2}\right)$ and realized by the iDE method of digital nanopatterning performed experimentally with an insertion loss lower than $\sim 1.55 \mathrm{~dB}$ and PERs better than $\sim 8 \mathrm{~dB}$ in a similar bandwidth. Hence, our topology-optimized PBS device is more compact and experimentally performs better.

\section{SUMMARY}

In summary, we have demonstrated that the inverse design engineering method of topology optimization can be utilized to optimize highly efficient, all-silicon, and broadband polarization beam splitters with $<4 \mu \mathrm{m}^{2}$ footprints. We find a classical trade-off between the device size and the performance. Utilizing the inverse design method of topology optimization it is possible to control the size of the device e.g. to comply with the required performance of the device and with the required minimum device packing density in the photonic circuit. Also, we find a trade-off between the smallest allowed feature sizes and the performance of the device. This reveals the power that lies in letting light scatter on a subwavelength scale. For purpose of experimental characterization, we fabricated a polarization beam splitter with a footprint of $\sim 2 \mu \mathrm{m}^{2}$ and demonstrated a demultiplexing loss lower than $\sim 0.82 \mathrm{~dB}$ and an extinction ratio larger than $\sim 15$ $\mathrm{dB}$ for both polarization modes in the C-band. Compared to a previous experimentally demonstrated polarization beam splitter our device is performing with lower loss and a higher extinction ratio in a similar bandwidth despite only taking up $\sim 35 \%$ of the space. To our knowledge, our device is the best performing and most compact all-silicon polarization beam splitter experimentally demonstrated to date for the C-band. We reckon that a set of design rules, e.g. minimum transmission efficiency and extinction ratio over a certain wavelength interval should be defined before conducting thorough systematic investigations to claim absolute compactness of photonic devices. However, our results further underline the potential of topology optimization to deliver high-performing and ultra-compact designs to future nanophotonic circuits. 


\section{REFERENCES}

[1] J.S. Jensen and O. Sigmund, "Topology optimization for nano-photonics", Laser \& Photon. Rev. 5(2), pp. 308$321(2011)$.

[2] J. Lu and J. Vǔcković, "Nanophotonic computational design," Opt. Express 21(11), pp. 13351-13367 (2013).

[3] A.Y. Piggott, J. Lu, K.G. Lagoudakis, J. Petykiewicz, T.M. Babinec and J. Vučković, "Inverse design and demonstration of a compact and broadband on-chip wavelength demultiplexer", Nature Photon. 9, pp. 374-377 (2015).

[4] B. Shen, P. Wang, R. Polson and Rajesh Menon, "An integrated-nanophotonics polarization beamsplitter with $2.4 \times 2.4 \mu \mathrm{m}^{2}$ footprint", Nature Photon. 9, pp. 378-382 (2015).

[5] M. P. Bendsøe and N. Kikuchi, "Generating optimal topologies in structural design using a homogenization method," Comput. Meth. Appl. Mech. Engng. 71(2), pp. 197-224 (1988).

[6] P.I. Borel, A. Harpøth, L.H. Frandsen, M. Kristensen, P. Shi, J.S. Jensen and O. Sigmund, "Topology optimization and fabrication of photonic crystal structures", Opt. Express 12(9), pp. 1996-2001 (2004).

[7] L.H. Frandsen, Y. Elesin, L.F. Frellsen, M. Mitrovic, Y. Ding, O. Sigmund and K. Yvind, "Topology optimized mode conversion in a photonic crystal waveguide fabricated in silicon-on-insulator material", Opt. Express 22(7), pp. 8525-8532 (2014).

[8] Louise F. Frellsen, Lars H. Frandsen, Yunhong Ding, Yuriy Elesin, Ole Sigmund and Kresten Yvind, “Topology-optimized silicon photonic wire mode (de)multiplexer", Proc. SPIE 9367, 9367-0X (2015).

[9] L.H. Frandsen, Y. Elesin, O. Sigmund, O., J.S. Jensen and K. Yvind, "Wavelength Selective 3D Topology Optimized Photonic Crystal Devices", in CLEO: 2013, OSA Technical Digest CTh4 L.6 (Optical Society of America, 2013).

[10] Lars H. Frandsen, Yuriy Elesin, Ole Sigmund, and Kresten Yvind, "Topology Optimization of Coupled Photonic Crystal Cavities for Flat-top Drop Filter Functionality," in CLEO/Europe-EQEC: 2015, OSA Technical Digest CK-9.1 (Optical Society of America, 2015).

[11] K. Yamada, T. Tsuchizawa, H. Fukuda, C. Koos, J. Pfeifle, J.H. Schmid, P. Cheben, P.J. Bock, and A.P. Knights, Handbook of Silicon Photonics (CRC Press, 2013), Chap. 2.

[12]X. Guan, H. Wu, Y. Shi, and D. Dai, "Extremely small polarization beam splitter based on a multimode interference coupler with a silicon hybrid plasmonic waveguide", Opt. Lett. 39(2), pp. 259-262 (2014).

[13] Y. Elesin, B.S. Lazarov, J.S. Jensen, and O. Sigmund, "Time domain topology optimization of 3D nanophotonic devices," Photon. Nanostruct. Fund. Appl. 12(1), pp. 23-33 (2014).

[14] Y. Elesin, B.S. Lazarov, J.S. Jensen and O. Sigmund, "Design of robust and efficient photonic switches using topology optimization", Photon. Nanostruct. Fund. Appl. 10(1), pp. 153-165 (2012). 